\title{
Docosahexaenoic Acid Augments Hypothermic Neuroprotection in a Neonatal Rat Asphyxia Model
}

\author{
Deborah R. Berman ${ }^{\mathrm{a}}$ Ellen Mozurkewich ${ }^{\mathrm{a}}$ Yiqing Liu ${ }^{\mathrm{b}}$ Yu Shangguan ${ }^{\mathrm{b}}$ \\ John D. Barks ${ }^{b}$ Faye S. Silverstein ${ }^{b, c}$ \\ ${ }^{\text {a }}$ Division of Maternal Fetal Medicine, Department of Obstetrics and Gynecology, and Departments of bediatrics \\ and 'Neurology, University of Michigan, Ann Arbor, Mich., USA
}

\section{Key Words}

Albumin · Docosahexaenoic acid · Hypothermia

\begin{abstract}
Background: In neonatal rats, early post-hypoxia-ischemia (HI) administration of the omega-3 fatty acid docosahexaenoic acid (DHA) improves sensorimotor function, but does not attenuate brain damage. Objective: To determine if DHA administration in addition to hypothermia, now standard care for neonatal asphyxial brain injury, attenuates post-HI damage and sensorimotor deficits. Methods: Seven-day-old (P7) rats underwent right carotid ligation followed by $90 \mathrm{~min}$ of $8 \% \mathrm{O}_{2}$ exposure. Fifteen minutes later, pups received injections of DHA $2.5 \mathrm{mg} / \mathrm{kg}$ (complexed to $25 \%$ albumin) or equal volumes of albumin. After a 1-hour recovery, pups were cooled $\left(3 \mathrm{~h}, 30^{\circ} \mathrm{C}\right)$. Sensorimotor and pathology outcomes were initially evaluated on P14. In subsequent experiments, sensorimotor function was evaluated on P14, P21, and P28; histopathology was assessed on P28. Results: At P14, left forepaw function scores (normal: 20/20) were near normal in DHA + hypothermia-treated animals (mean \pm SD $19.7 \pm 0.7 \mathrm{DHA}+$ hypothermia vs. $12.7 \pm 3.5$ albumin + hypothermia, $\mathrm{p}<0.0001$ ) and brain damage was reduced (mean \pm SD right hemisphere damage $38 \pm 17 \%$ with $\mathrm{DHA}+$ hypo-
\end{abstract}

\section{KARGER}

(c) 2013 S. Karger AG, Base

$1661-7800 / 13 / 1041-0071 \$ 38.00 / 0$

E-Mail karger@karger.com

www.karger.com/neo thermia vs. $56 \pm 15 \%$ with albumin + hypothermia, $p=0.003$ ). Substantial improvements on three sensorimotor function measures and reduced brain damage were evident up to P28. Conclusion: Unlike post-HI treatment with DHA alone, treatment with DHA + hypothermia produced both sustained functional improvement and reduced brain damage after neonatal $\mathrm{HI}$.

Copyright $\odot 2013$ S. Karger AG, Basel

\section{Introduction}

Docosahexaenoic acid (DHA) is a dietary long-chain omega-3 polyunsaturated fatty acid with neuroprotective properties [1-7]. DHA modulates neuroinflammation, oxidative stress, and apoptosis [7-9]. We previously reported that DHA pretreatment is neuroprotective in a neonatal rodent model of hypoxia-ischemia (HI) brain injury, elicited by right carotid artery ligation and timed exposure to $8 \% \mathrm{O}_{2}$ in 7-day-old (P7) rats [4]; a single injection of DHA prior to lesioning improved sensorimotor function and reduced brain damage. Sub-

D.R.B. and E.M. contributed equally to this work. 
sequently, we found that a single DHA injection after the end of hypoxia exposure improved the same neurologic function, but did not reduce tissue injury [5]. Other investigators have shown that chronic maternal dietary supplementation with DHA or fish oil (enriched in DHA) in the prenatal and lactation period also confers neuroprotection and reduces brain damage in their offspring in this model $[6,7]$. Since most perinatal asphyxia insults occur sporadically and unpredictably in term infants, we focused our preclinical investigations on optimization of the efficacy of DHA administered after acute $\mathrm{HI}$ injury.

Hypothermia decreases death or disability in neonates with HI brain injury [10]. Yet, $40-50 \%$ of infants treated with hypothermia have adverse outcomes and treatments to augment the neuroprotective efficacy of hypothermia are needed [11]. Potentially neuroprotective drugs, such as DHA, administered in conjunction with therapeutic hypothermia, could exert additive or synergistic neuroprotection or paradoxically counteract the benefits of cooling. To further evaluate the neuroprotective properties of DHA, it was thus essential to examine its efficacy in combination with hypothermia. We developed a protocol for delayed onset, relatively brief postinjury cooling to evaluate drug-hypothermia interactions, either beneficial or harmful, in the neonatal rodent HI brain injury model [12-14]. The stepwise evaluation begins with a comparison of drug versus vehicle and then comparison of adding hypothermia to drug- and vehicle-treated groups. Based on our prior report [5], in this study, we selected a post-HI DHA dose that resulted in better sensorimotor function than in vehicle-treated controls without any benefit on tissue damage. We examined the impact of combining this DHA dose with brief hypothermia on function and neuropathology outcomes. We found sustained benefits of combination treatment with DHA and brief hypothermia.

\section{Methods}

DHA Treatment

DHA (Sigma-Aldrich, St. Louis, Mo., USA) was complexed to human $25 \%$ albumin, as previously described (final concentration $0.5 \mathrm{mg} / 1 \mathrm{ml}$ ) [4]. The dose selected, $2.5 \mathrm{mg} / \mathrm{kg}$, was more effective than either higher $(5 \mathrm{mg} / \mathrm{kg})$ or lower $(1 \mathrm{mg} / \mathrm{kg})$ doses in the postHI treatment protocol reported previously [5].

Wistar rat pups were obtained in litters adjusted to equal sex distribution (Charles River Laboratories, Portage, Mich., USA) and were weaned on P21. Animals were treated in accordance with protocols approved by the University of Michigan Committee on the Use and Care of Animals.
Each of six independent experiments ( $n=12$ animals/experiment) included equal numbers of littermate DHA-treated and albumin-treated pups; animals of both genders were allocated equally between groups in each experiment.

Isoflurane-anesthetized pups underwent right carotid artery ligation, as previously described $[4,15]$. Pups recovered $\left(36.5^{\circ} \mathrm{C}, 90\right.$ $\mathrm{min})$ and were then exposed to $8 \% \mathrm{O}_{2}(90 \mathrm{~min})$. After 15 -min recovery periods (in $37^{\circ} \mathrm{C}$ incubators), they received equal-volume intraperitoneal injections $(0.05 \mathrm{ml} / 10 \mathrm{~g})$ of DHA or $25 \%$ albumin; they were then returned to incubators ( $45 \mathrm{~min}$ ) until initiation of hypothermia. Pups were then placed in a circulating air incubator with cuffed portals $\left(30^{\circ} \mathrm{C}, 3 \mathrm{~h}\right)$ and were separated with partitions to prevent huddling $[13,14]$. Pups were then returned to dams until P14 (3 experiments) or P28 (3 experiments). Seven sequential temperature measurements were obtained (YSI thermometer 43T with probe 554; YSI Inc., Yellow Springs, Ohio, USA) from baseline to the conclusion of hypothermia [14].

\section{Sensorimotor Testing}

In this $\mathrm{HI}$ model, a variety of sensorimotor tests provide useful outcome measures in neuroprotection studies $[4,5,7,12-14,16$, 17]. We incorporated three quantifiable, objective tests.

Vibrissae-Stimulated Forepaw Place Testing. On P14, or weekly on $\mathrm{P} 14, \mathrm{P} 21$, and $\mathrm{P} 28$, animals underwent lateral vibrissae-stimulated forepaw place testing $[4,5]$. Vibrissae are stimulated unilaterally on a surface edge. As early as P14, the normal complete response is consistent immediate extension of the ipsilateral forepaw to contact the stimulus surface. Performance is impaired in the forepaw contralateral to an HI cerebral hemisphere lesion. The impaired forepaw either does not move or demonstrates a partial extension movement that is insufficient to reach the stimulus surface. Performance was videotaped and scored in 10 trials/forepaw by an observer unaware of treatment group (partial response score $=1$, complete response score $=2$ ) [4].

Grip Traction Test. On P21 and P28, forepaw grip strength (maximal force applied in grasping a pull bar) was measured using a Grip Strength Meter (3 measurements/forepaw; Columbus Instruments, Columbus, Ohio, USA). Normal animals have equal strength bilaterally, and grip strength increases between P21 and P28 [14]. Absolute values for grip strength and left/right forepaw grip strength ratios were calculated for each animal. After HI lesioning for 90 min on $\mathrm{P} 7$, animals typically have about $40-50 \%$ reduction in contralateral grip strength 2-4 weeks later [14].

Vertical Cylinder Exploration. On P28, forepaw preference was evaluated. Normal animals initiate exploratory movements equally with both forepaws; animals that underwent HI lesioning on P7 typically display decreased initiation of weight-bearing contacts with the forepaw contralateral to brain injury [16]. Animals were videotaped in a vertical clear plastic cylinder $(20 \times 30 \mathrm{~cm}, 2 \mathrm{~min})$, and initial weight-bearing contacts of the right, left, or both forepaws with the cylinder wall were counted. Right forepaw preference scores were calculated with the formula: $100 \times($ right - left $) /$ $($ right + left + both $)($ normal $=0)[18]$.

\section{Histopathology}

Animals were euthanized and brains removed and frozen on P14 or P28. Regularly spaced coronal $20-\mu \mathrm{m}$ sections, from the anterior genu to the posterior genu of the corpus callosum, were cresyl violet stained. Using ImageJ software (US National Institutes of Health, Bethesda, Md., USA; http://rsb.info.nih.gov/ij/), 
Table 1. Sequential temperature measurements

\begin{tabular}{lllllllll}
\hline Experiment/group & $\mathrm{n}$ & \multicolumn{6}{l}{ Time-points } \\
\cline { 2 - 9 } & & $\begin{array}{l}1 \\
\text { presurgery }\end{array}$ & $\begin{array}{l}2 \\
\text { end of HI }\end{array}$ & $\begin{array}{l}3 \\
15 \text { min post-HI }\end{array}$ & 60 min post-HI & 75 min post-HI & 90 min post-HI & 240 min post-HI \\
\hline DHA + HT & 36 & $33.1 \pm 0.8$ & $35.0 \pm 0.6$ & $35.2 \pm 0.5$ & $36.0 \pm 0.4$ & $31.3 \pm 0.8$ & $31.2 \pm 0.7$ & $32.2 \pm 1.0$ \\
ALB + HT & 36 & $33.3 \pm 0.8$ & $34.8 \pm 0.7$ & $35.3 \pm 0.6$ & $36.1 \pm 0.3$ & $31.3 \pm 0.8$ & $31.1 \pm 0.6$ & $32.2 \pm 1.1$ \\
\hline
\end{tabular}

Temperatures $\left({ }^{\circ} \mathrm{C}\right)$ are expressed as means \pm SD. All animals underwent HI lesioning (see Methods); 15 min after the end of $\mathrm{HI}$, all received injections of DHA $2.5 \mathrm{mg} / \mathrm{kg}$ (in $25 \%$ albumin) or an equivalent volume of $25 \%$ albumin. All animals underwent hypothermia $\left(30^{\circ} \mathrm{C}\right.$ incubator for $3 \mathrm{~h}$, beginning at $1 \mathrm{~h}$ after $\left.\mathrm{HI}\right)$; time-point 4 corresponds with the beginning of hypothermia and time-point 7 is at the end of hypothermia. Data presented includes 3 litters with outcome to P14 and 3 litters with outcome to P28. HT = Hypothermia; $\mathrm{ALB}=25 \%$ albumin.

bilateral volumes were calculated from hemisphere and regional area measurements in at least 10 sections/brain; percent reductions in right hemisphere volumes, compared to left, were calculated with the formula: $100 \times($ left - right/left) [14].

\section{Statistics}

Sample sizes (18/group) were selected based on power calculations with data from prior similar experiments. Differences in contralateral vibrissae-stimulated forepaw placing responses, forepaw grip strength, and cylinder right forepaw preference score were compared using repeated measures ANOVA. Post hoc comparisons of treatment group means were carried out using the TukeyKramer single-step multiple comparison procedure. A linear mixed models ANOVA was applied to evaluate differences in percent brain damage. We used litter as a random effect, with treatment, sex, and brain region as fixed effects. Differences in body temperatures and weights were assessed by repeated measures ANOVA, factoring treatment and sex.

\section{Results}

\section{Survival/Morbidity}

There were no differences in mortality, mean temperatures (table 1), or weights or weight changes (not shown).

\section{Initial (P14) Outcomes}

Sensorimotor Function. Right forepaw placing scores were normal in all DHA-treated and 16 of 17 albumintreated animals (one scored 18/20). Contralateral (left) placing responses were near normal in the DHA + hypothermia group, but significantly impaired in the albumin + hypothermia group (total scores: $19.7 / 20 \pm 3.5 \mathrm{vs.} 12.7 / 20$ $\pm 0.7, \mathrm{p}<0.0001$, t test; complete responses: $9.7 / 10 \pm 0.6 \mathrm{vs}$. $5.9 / 10 \pm 1.8, \mathrm{p}<0.0001$, t test; no gender effect; fig. 1$)$.

Histopathology. In controls, there was $55 \pm 15 \%$ right hemisphere volume loss; damage was modestly attenu-

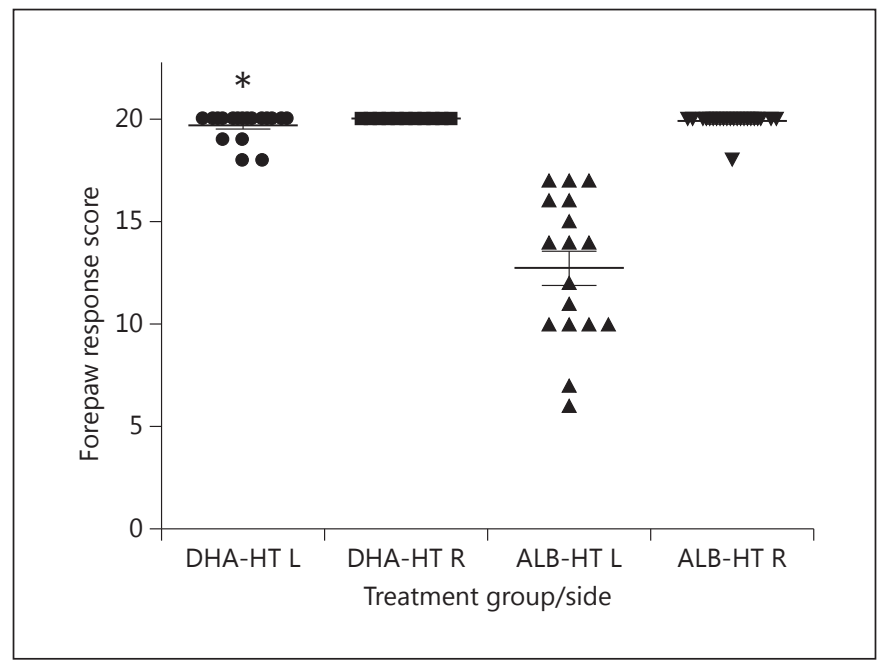

Fig. 1. Treatment with DHA and delayed-onset hypothermia attenuates P14 forepaw placing deficits. P14 bilateral vibrissae-stimulated forepaw placing test scores (mean \pm SEM, normal score $=20$; see Methods) in animals that underwent right carotid ligation followed by 90 min $8 \% \mathrm{O}_{2}$ exposure on P7, and received DHA, $2.5 \mathrm{mg}$ / $\mathrm{kg}$, or $25 \%$ albumin, followed $1 \mathrm{~h}$ later by hypothermia $\left(3 \mathrm{~h}, 30^{\circ} \mathrm{C}\right)$. Left (L) forepaw placement was impaired in albumin-treated controls $(\mathrm{n}=17$, ALB HT L), but performance was near-normal in the DHA-HT group $\left(\mathrm{n}=18\right.$, DHA HT L, ${ }^{*} \mathrm{p}<0.01$ ANOVA, TukeyKramer post hoc test).

ated in the DHA + hypothermia group $(38 \pm 17 \%$, p $=$ 0.003 , t test; fig. 2). Figure $3 \mathrm{a}$, c illustrates a representative control lesion with right striatal and hippocampal atrophy and cortical infarction. Figure $3 b$, d illustrates representative pathology from the DHA + hypothermia group with greater tissue preservation. Table 2 presents regional volume data. On P14, tissue damage was reduced in the 
Fig. 2. Treatment with DHA and hypothermia reduces P14 brain damage. Bilateral cerebral hemisphere volumes on P14, calculated from cross-sectional area measurements (see Methods), are compared in the DHA + hypothermia (DHA-HT, $\mathrm{n}=18$ ) and albumin + hypothermia (ALB-HT, $\mathrm{n}=17$ ) groups (box: median and interquartile range; whiskers: minimum and maximum). Right hemisphere damage is reduced in the DHA + hypothermia-treated group ( ${ }^{*} \mathrm{p}<0.05$, t test). $\mathrm{L}=$ Left; $\mathrm{R}=$ right; HEM $=$ hemisphere.

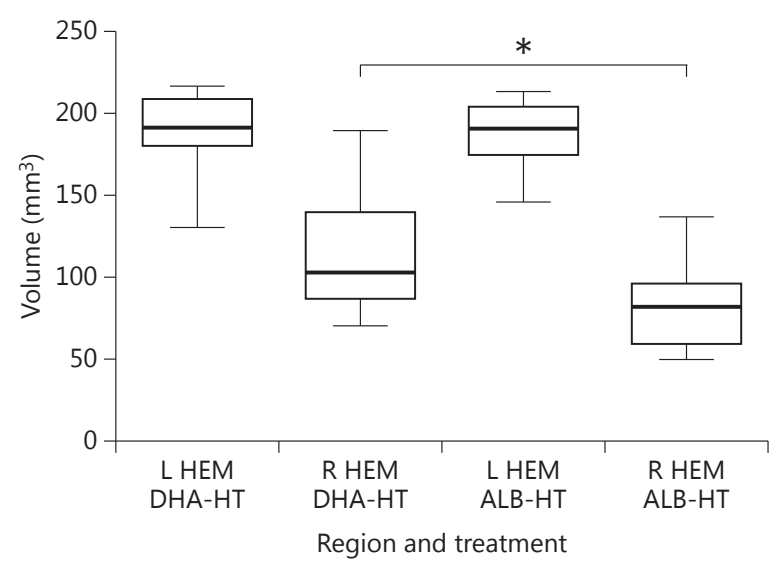

Fig. 3. Neuropathology. These cresyl violet-stained coronal sections at the level of the striatum $(\mathbf{a}, \mathbf{b})$ and hippocampus $(\mathbf{c}, \mathbf{d})$ illustrate representative histopathology in DHA + hypothermia- (b, d) and albumin + hypothermia- $(\mathbf{a}, \mathbf{c})$ treated animals on $\mathrm{P} 14$. After right carotid ligation $+90 \mathrm{~min}$ $8 \% \mathrm{O}_{2}$ exposure, $\mathrm{P} 7$ pups received DHA, $2.5 \mathrm{mg} / \mathrm{kg}$ (b, d), or $25 \%$ albumin vehicle, followed $1 \mathrm{~h}$ later by hypothermia $(3 \mathrm{~h}$, $30^{\circ} \mathrm{C}$ ). In the control (a, c), there is right cortical infarction (arrowheads), striatal atrophy and infarction $(*)$, and hippocampal atrophy (arrow). In the DHA + hypothermia brain (b, d), there is less right hemisphere, striatal $\left(^{*}\right)$, and hippocampal (arrow) atrophy (scale bar $=1 \mathrm{~mm}$ ).
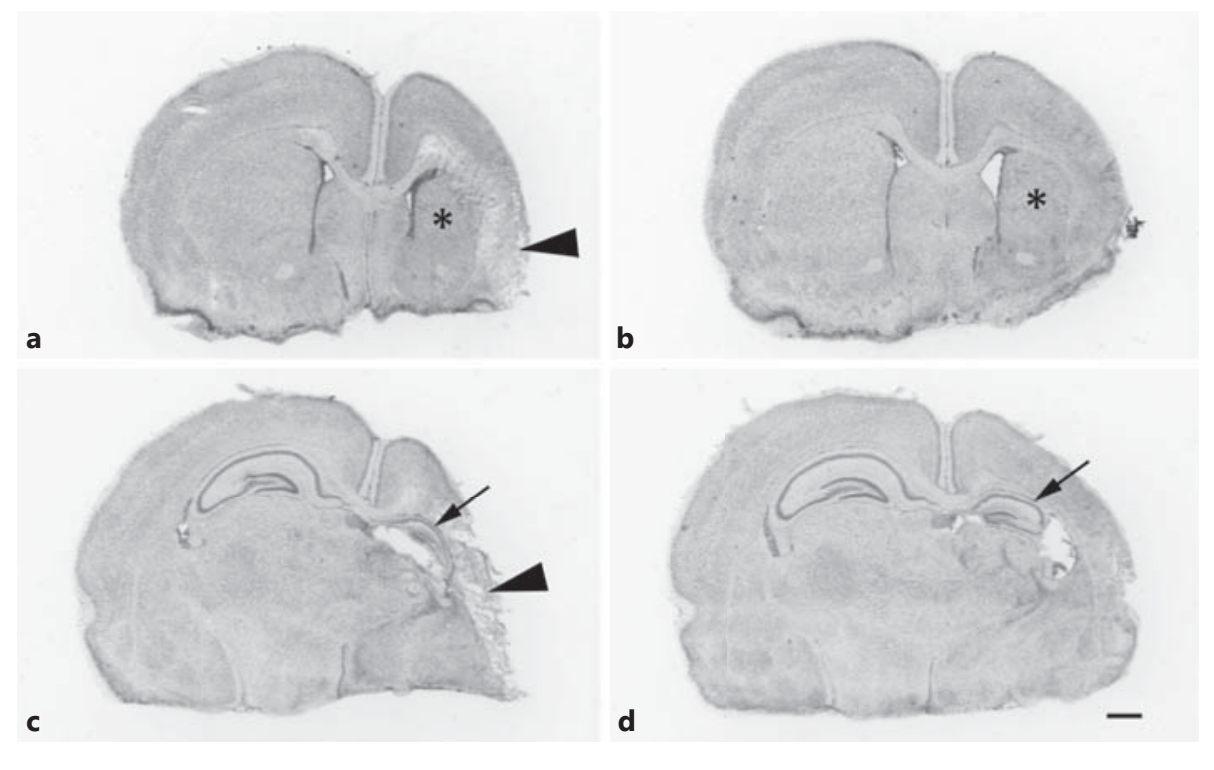

Table 2. Effect of DHA and therapeutic hypothermia (HT) on regional brain damage severity

\begin{tabular}{|c|c|c|c|c|c|c|c|c|c|c|}
\hline \multirow[t]{2}{*}{ Treatment } & \multirow[t]{2}{*}{$\mathrm{n}$} & \multicolumn{3}{|l|}{ Cortex } & \multicolumn{3}{|c|}{ Striatum } & \multicolumn{3}{|c|}{ Hippocampus } \\
\hline & & left & right & $\%$ diff. & left & right & $\%$ diff. & left & right & $\%$ diff. \\
\hline \multicolumn{11}{|l|}{ P14 outcomes } \\
\hline $25 \%$ albumin $+\mathrm{HT}$ & 17 & $69 \pm 16$ & $25 \pm 15$ & $67 \pm 11$ & $23 \pm 4$ & $13 \pm 4$ & $46 \pm 3$ & $6 \pm 3$ & $2 \pm 1$ & $64 \pm 1$ \\
\hline DHA $2.5 \mathrm{mg} / \mathrm{kg}+\mathrm{HT}$ & 18 & $62 \pm 16$ & $42 \pm 19^{*}$ & $40 \pm 13^{*}$ & $23 \pm 4$ & $16 \pm 5^{*}$ & $32 \pm 3^{*}$ & $6 \pm 3$ & $2 \pm 1$ & $61 \pm 1$ \\
\hline \multicolumn{11}{|l|}{ P28 outcomes } \\
\hline $25 \%$ albumin $+\mathrm{HT}$ & 17 & $98 \pm 7$ & $29 \pm 13$ & $70 \pm 13$ & $34 \pm 4$ & $13 \pm 3$ & $62 \pm 10$ & $13 \pm 3$ & $2 \pm 2$ & $83 \pm 13$ \\
\hline $\mathrm{DHA} 2.5 \mathrm{mg} / \mathrm{kg}+\mathrm{HT}$ & 18 & $98 \pm 8$ & $41 \pm 18^{*}$ & $59 \pm 17^{*}$ & $34 \pm 3$ & $17 \pm 5^{*}$ & $50 \pm 13^{*}$ & $13 \pm 3$ & $4 \pm 3^{*}$ & $68 \pm 18^{*}$ \\
\hline
\end{tabular}

All animals were lesioned on P7 and tissue damage was evaluated on P14 or P28 (see Methods). Left and right values represent regional volumes in each cerebral hemisphere $\left(\mathrm{mm}^{3}\right.$, mean $\left.\pm \mathrm{SD}\right) . \%$ difference values (mean $\left.\pm \mathrm{SD}\right)$ represent regional \% damage, calculated from regional volumes with the formula: $100 \times($ left - right $) /$ left. diff. $=$ Difference. ${ }^{*} \mathrm{p}<0.05$ compared to albumin $+\mathrm{HT}($ ANOVA with Tukey-Kramer post hoc test). 


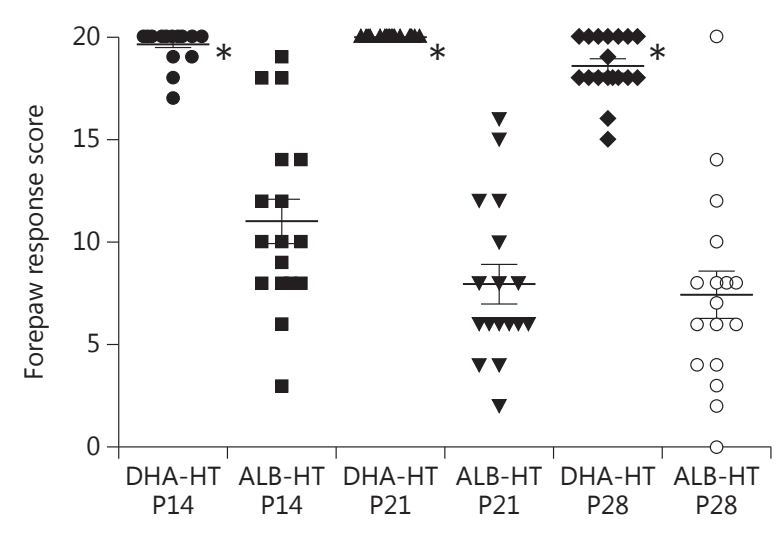

a

Treatment and age
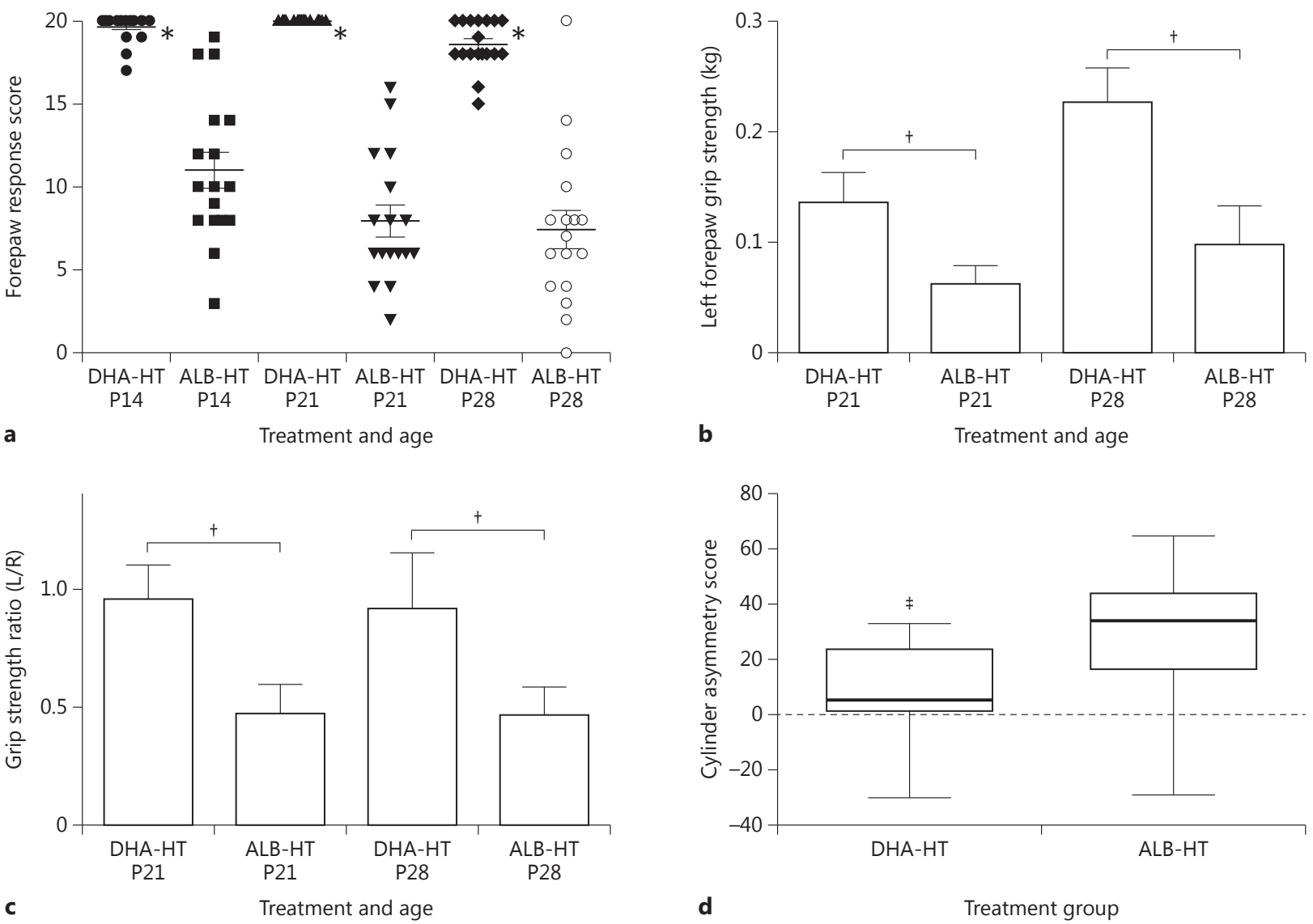

Fig. 4. Performance on three measures of sensorimotor function. Vibrissae-stimulated forepaw placing was evaluated on P14, P21, and P28 (a); forepaw grip strength on P21 and P28 (b, c) and vertical cylinder forepaw contact preference on P28 (d) in the DHA + hypothermia (DHA-HT) and control albumin + hypothermia (ALB-HT) groups. a Left forepaw vibrissae-stimulated placing scores (mean \pm SEM) remained near normal in the DHA + HT group $(n=18)$, and impaired placing persisted in the ALB + HT controls ( $\mathrm{n}=17 ;^{*} \mathrm{p}<0.0001$ for treatment, $\mathrm{p}<0.01$ for time, with no treatment-time interaction, repeated-measures ANOVA). b Left forepaw grip strength (mean \pm SD) increased from P21 to P28 ( $p<0.0001$, repeated-measures ANOVA). In the DHA-HT

group, there was no right-left difference at P21 or P28 (c); in ALBHT controls, left forepaw grip strength was reduced at both ages compared to right forepaw grip strength (mean \pm SD L/R ratio) (c) and compared to left forepaw grip strength in the DHA + hypothermia group $\left({ }^{\dagger} \mathrm{p}<0.0001, \mathrm{ANOVA}\right)(\mathbf{b}, \mathbf{c})$, and remained at about $50 \%$ of right forepaw strength at both ages (L/R grip strength ratio, ${ }^{\dagger} \mathrm{p}<0.0001$ for treatment, repeated-measures ANOVA) (c). d In the vertical cylinder test, on P28, both groups had positive scores, indicating right forepaw preference (box: median and interquartile range; whiskers: minimum and maximum), but asymmetry was attenuated (i.e. lower score) in the DHA-HT group $\left({ }^{\ddagger} \mathrm{p}<0.005\right.$, t test).

DHA + hypothermia group (ANOVA, p $<0.001)$ with region-specific treatment effects in the cortex $(\mathrm{p}=0.0004)$ and striatum $(\mathrm{p}=0.0014)$, and no gender effects.

\section{Outcomes from P14 to P28}

Sensorimotor Function. All right forepaw placing scores were normal. Left forepaw scores remained near normal in the DHA + hypothermia group, and deficits persisted in the albumin + hypothermia group $(\mathrm{p}<0.0001$ for treatment, $\mathrm{p}<0.01$ for time, with no treatment-time interaction, repeated-measures ANOVA; fig. 4a). Right forepaw grip strength increased from P21 to P28 (fig. 4b). In the DHA + hypothermia group, left and right forepaw grip strength did not differ; in the albumin + hypothermia group, left forepaw strength remained at about $50 \%$ of right forepaw grip strength (grip strength ratio, $\mathrm{p}<0.0001$ 


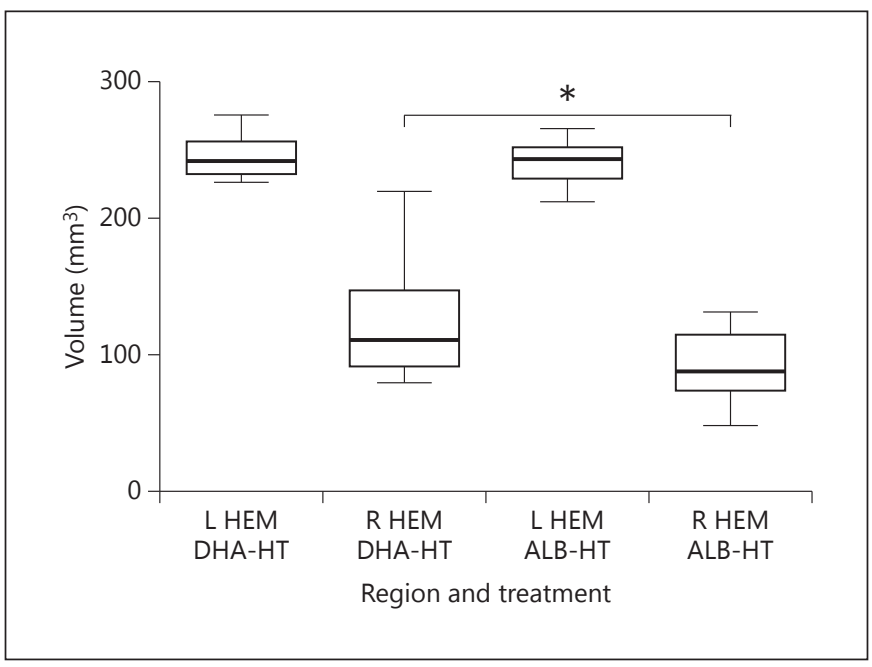

Fig. 5. Sustained attenuation of brain damage by DHA + hypothermia. P28 bilateral cerebral hemisphere volumes were compared in the DHA + hypothermia (DHA-HT, $\mathrm{n}=18$ ) and albumin + hypothermia (ALB-HT, $\mathrm{n}=17$ ) groups (box: median and interquartile range; whiskers: minimum and maximum). Right hemisphere damage is less severe in the DHA + hypothermia group $\left({ }^{*} \mathrm{p}<0.05\right)$. $\mathrm{L}=$ Left; $\mathrm{R}=$ right; HEM = hemisphere.

for treatment, $\mathrm{p}=\mathrm{NS}$ for time, repeated-measures ANO$\mathrm{VA}$; fig. 4c). In the cylinder test, at P28 there were asymmetries in both groups; asymmetric function was greater in the albumin + hypothermia than DHA + hypothermia groups ( $\mathrm{p}<0.005$, $\mathrm{t}$ test; fig. $4 \mathrm{~d})$. There were no gender effects on any scores.

Histopathology. Results were congruent with P14 outcomes (fig. 5). In the DHA + hypothermia group, there were region-specific effects in the cortex, striatum, and hippocampus ( $\mathrm{p}<0.05$ ANOVA; $\mathrm{p}<0.05$, Tukey-Kramer post hoc tests). There were no gender effects.

\section{Discussion}

Our results demonstrate that the combination of postHI treatment with DHA and brief delayed hypothermia confers markedly improved sensorimotor function and modestly reduced tissue injury, compared to treatment with delayed hypothermia without DHA.

Brief post-HI hypothermia (started immediately after hypoxia) may delay the progression of brain injury without conferring sustained neuroprotection [19]. Therefore, we replicated the P14 outcome experiments and confirmed that the beneficial effects of DHA + hypother- mia treatment were sustained. In addition, we incorporated three complementary sensorimotor tests to further characterize functional outcomes and to confirm the persistence of improvements. Each testing method included objective, quantifiable measures. All three measures demonstrated substantially better function in the DHA + hypothermia group; persistent asymmetries were most evident in the cylinder test. This task may be more sensitive to detection of subtle sensorimotor deficits.

Although this brief duration of hypothermia is not equivalent to the much longer duration of hypothermia that is currently applied clinically, our data provide important proof-of-principle evidence that the combination of DHA with hypothermia could represent a more effective postasphyxia treatment than hypothermia alone. In an adult rat focal cerebral ischemia with reperfusion model, postreperfusion treatment with a fatty acid mixture that included DHA aggravated injury, presumably by increasing the ischemia-induced oxidative burden [20]. Thus, in terms of translational potential for DHA in neonates, it is reassuring that we found no adverse interactions between DHA and hypothermia. The finding that DHA + hypothermia improved outcomes in a neonatal rodent model provides the impetus for more complex and costly experiments in larger neonatal animal models (e.g. piglets) in which prolonged hypothermia and physiologic monitoring are feasible. Future experiments could also clarify whether combination treatment conferred additive or synergistic neuroprotection.

The current study has several additional limitations. Although inclusion of a DHA-no hypothermia control group would have been ideal, we employed a pragmatic design that has been used to evaluate other novel therapeutic agents in combination with hypothermia $[13,14]$. We prioritized comparisons with littermate controls and evaluation of gender effects. DHA was complexed to albumin; although higher albumin doses may have neuroprotective properties, we previously showed that the albumin doses and administration route used in these experiments had no effects on outcomes in comparison with saline-injected controls [4].

Hypothermia could, hypothetically, either attenuate or amplify DHA neuroprotection. Hypothermia could result in increased accumulation of DHA-derived toxic isoprostanes and/or impaired conversion of DHA to its neuroprotective metabolite $[8,9]$; however, we found no detrimental interactions. We speculate that in the injured neonatal brain, hypothermia increased the neuroprotective efficacy of DHA by reducing nonenzymatic DHA peroxidation [21-23] and/or by preserving DHA for en- 
zymatic conversion via 15-lipoxygenase to the reported neuroprotective metabolite 10,17S-docosatriene (neuroprotectin D1) [9]. In an adult rat acute hypoxia model, hypothermia reduced lipid peroxidation [21]. We did not explore the underlying cellular and molecular mechanisms whereby DHA and hypothermia act in concert to attenuate brain injury and preserve sensorimotor function; this is a complex and challenging goal, and beyond the scope of this proof-of-principle study.

One of the intriguing findings at P28 was the nearnormal sensorimotor function in the DHA + hypothermia combination group, despite substantial forebrain tissue damage. This could suggest that DHA and hypothermia together promote plasticity mechanisms during recovery, e.g. increased production of neurotrophins and/or stimulation of neurogenesis. In adult brain after global cerebral ischemia, postresuscitation hypothermia augments brain-derived neurotrophic factor expression [24], and in normal adult rats a diet enriched in DHA increased brain-derived neurotrophic factor expression in brain (the time course for this response was not examined) [25]. Similarly, both interventions may also increase neurogenesis and could contribute to promotion of recovery, in part, through this mechanism [26, 27].

Several drugs, including DHA, are attractive candidates for post-HI therapy in combination with hypothermia [11]. It will be challenging to decide which agents should move forward to clinical trial assessment. DHA readily crosses the blood-brain barrier, is designated Generally Regarded as Safe (GRAS) by the United States Food and Drug Administration [28], and can be administered parenterally. An investigational omega-3-enriched parenteral lipid emulsion is currently under study in neonates [29], and additional safety data is likely to emerge. The dose of DHA tested, $2.5 \mathrm{mg} / \mathrm{kg}$, is within the feasible range for clinical applications with this emulsion.

\section{Conclusions}

Our findings provide the impetus for future studies to determine if findings with DHA + hypothermia can be replicated in large animal preclinical models of neonatal brain injury. The optimal dose(s) and routes of administration of DHA for postischemic neuroprotection also remain to be determined. Confirmatory results from additional preclinical studies could provide a compelling rationale for initiation of randomized controlled trials to test the effectiveness of DHA in combination with therapeutic hypothermia in neonates with moderate-to-severe hypoxic-ischemic encephalopathy.

\section{Acknowledgements}

This work was supported through the NIH/NCRR CTSA grant UL1 RR024986, by NIH grant R21 AT006636 (to J.D.B. and F.S.S.), by a Holden Research Award (to J.D.B.) and by the GorgEffen Gift Fund of the Department of Pediatrics, The University of Michigan.

\section{Disclosure Statement}

D.R.B., Y.L., F.S.S., and Y.S. have no conflicts of interest to disclose. E.M. is the principal investigator on an NIH-sponsored randomized controlled trial that has received donated fish oil capsules and placebo capsules from Nordic Naturals, Watsonville, Calif., USA. J.D.B. received a donation of DHASCO oil from Martek Biosciences Inc., Columbia, Md., USA, for a different rodent research project. The funding sources played no role in study design, in the collection, analysis and interpretation of data, in the writing of the report, or in the decision to submit the article for publication.

\section{References}

1 Belayev L, Marcheselli VL, Khoutorova L, Rodriguez de Turco EB, Busto R, Ginsberg MD, Bazan NG: Docosahexaenoic acid complexed to albumin elicits high-grade ischemic neuroprotection. Stroke 2005;36:118-123.

-2 Huang WL, King VR, Curran OE, Dyall SC, Ward RE, Lal N, Priestly JV, Michael-Titus AT: A combination of intravenous and dietary docosahexaenoic acid significantly improves outcome after spinal cord injury. Brain 2007;130:3004-3019.

-3 Pan H, Kao T, Ou Y, Yang DY, Yen YJ, Wang CC, Chuang YH, Liao SL, Raung SL, Wu CW,
Chiang AN, Chen CJ: Protective effect of docosahexaenoic acid against brain injury in ischemic rats. J Nutr Biochem 2009;20:715725.

4 Berman DR, Mozurkewich E, Liu Y, Barks J: Docosahexaenoic acid pretreatment confers neuroprotection in a rat model of perinatal cerebral hypoxia-ischemia. Am J Obstet Gynecol 2009;200:305.e1-e6.

5 Berman DR, Liu Y, Barks J, Mozurkewich E: Treatment with docosahexaenoic acid after hypoxia-ischemia improves forepaw placing in a rat model of perinatal hypoxia-is- chemia. Am J Obstet Gynecol 2010;203: 385.e1-e5.

-6 Suganuma H, Arai Y, Kitamura Y, Hayashi M, Okumura A, Shimizu T: Maternal docosahexaenoic acid-enriched diet prevents neonatal brain injury. Neuropathology 2010;30: 597-605.

7 Zhang W, Hu X, Yang W, Gao Y, Chen J: Omega-3 polyunsaturated fatty acid supplementation confers long-term neuroprotection against neonatal hypoxic-ischemic brain injury through anti-inflammatory actions. Stroke 2010;41:2341-2347.
DHA Augments Hypothermic

Neuroprotection
Neonatology 2013;104:71-78 DOI: $10.1159 / 000351011$ 
$>_{8}$ Marcheselli V, Hong S, Lukiw W, Tian XH, Gronert K, Musto A, Hardy M, Gimenez JM, Chiang N, Serhan CN, Bazan NG: Novel docosanoids inhibit brain ischemia-reperfusion-mediated leukocyte infiltration and proinflammatory gene expression. J Biol Chem 2003;278:43807-43817.

-9 Bazan NG: Neuroprotectin D1-mediated anti-inflammatory and survival signaling in stroke, retinal degenerations and Alzheimer's disease. J Lipid Res 2009;50(suppl):S400S405.

10 Edwards AD, Brocklehurst P, Gunn AJ, Halliday $H$, Juszczak E, Levene M, Strohm B, Thoresen M, Whitelaw, A, Azzopardi D: Neurological outcomes at 18 months of age after moderate hypothermia for perinatal hypoxic ischaemic encephalopathy: synthesis and meta-analysis of trial data. BMJ 2010;340: c363.

11 Cilio MR, Ferriero DM: Synergistic neuroprotective therapies with hypothermia. Semin Fetal Neonat Med 2010;15:293-298.

12 Liu YQ, Barks JD, Xu G, Silverstein FS: Topiramate extends the therapeutic window for hypothermia-mediated neuroprotection after stroke in neonatal rats. Stroke 2004;35:14601465.

13 Barks JD, Liu YQ, Shangguan Y, Silverstein FS: Phenobarbital augments hypothermic neuroprotection. Pediatr Res 2010;67:532537.

14 Liu Y, Shangguan Y, Barks JD, Silverstein FS: Bumetanide augments the neuroprotective efficacy of phenobarbital plus hypothermia in a neonatal hypoxia-ischemia model. Pediatr Res 2012;71:559-565.

15 Rice JE, Vannucci RC, Brierley JB: The influence of immaturity on hypoxic-ischemic brain damage in the rat. Ann Neurol 1981;9: 131-141.
6 Grow JL, Liu YQ, Barks JD: Can lateralizing sensorimotor deficits be identified after neonatal cerebral hypoxia-ischemia in rats? Dev Neurosci 2003;25:394-402.

-17 Pazaiti A, Soubasi V, Spandou E, Karkavelas G, Georgiou T, Karalis P, Guiba-Tziampiri: Evaluation of long-lasting sensorimotor consequences following neonatal hypoxic-ischemic brain injury in rats: the neuroprotective role of MgSO4. Neonatology 2009;95:33-40.

18 Schallert T, Fleming SM, Leasure JL, Tillerson JL, Bland ST: CNS plasticity and assessment of forelimb sensorimotor outcome in unilateral rat models of stroke, cortical ablation, parkinsonism and spinal cord injury. Neuropharmacology 2000;39:777-787.

19 Trescher WH, Ishiwa S, Johnston MV: Brief post-hypoxic-ischemic hypothermia markedly delays neonatal brain injury. Brain Dev 1997; 19:326-338

20 Yang Y, Pan HC, Yen YJ, Wang CC, Chuang YH, Chen SY, Lin SY, Liao SL, Raung SL, Wu CW, Chou MC, Chiang AN, Chen CJ: Detrimental effects of post-treatment with fatty acids on brain injury in ischemic rats. Neurotoxicology 2007;28:1220-1229.

21 Alva N, Carbonell T, Palomeque J: Hypothermic protection in an acute hypoxia model in rats: acid-base and oxidant/antioxidant profiles. Resuscitation 2010;81;609-616.

22 Roberts LJ, Montine TJ, Markesbery WR, Tapper AR, Hardy P, Chemtob S, Dettbarn WD, Morrow JD: Formation of isoprostanelike compounds (neuroprostanes) in vivo from docosahexaenoic acid. J Biol Chem 1998;273:13605-13612.

-23 Signorini C, Ciccoli L, Leoncini S, Carloni S, Perrone S, Comporti M, Balduini M, Buonocore G: Free iron, total F-isoprostanes and total F-neuroprostanes in a model of neonatal hypoxic-ischemic encephalopathy: neuroprotective effect of melatonin. J Pineal Res 2009;46:148-154.
-24 D’Cruz BJ, Fertig KC, Filiano AJ, Hicks SD, DeFranco DB, Callaway CW: Hypothermic reperfusion after cardiac arrest augments brain-derived neurotrophic factor activation. J Cereb Blood Flow Metab 2002;22:843-851.

25 Wu A, Ying Z, Gomez-Pinilla F: Docosahexaenoic acid dietary supplementation enhances the effects of exercise on synaptic plasticity and cognition. Neuroscience 2008; 155 : 751-759.

$26 \mathrm{He}$ C, Qu X, Cui L, Wang J, Kang JX: Improved spatial learning performance of fat- 1 mice is associated with enhanced neurogenesis and neuritogenesis by docosahexaenoic acid. Proc Natl Acad Sci USA 2009;106: 11370-11375.

27 Xiong M, Cheng GQ, Ma SM, Yang Y, Shao XM, Zhou WH: Post-ischemic hypothermia promotes generation of neural cells and reduces apoptosis by $\mathrm{Bcl}-2$ in the striatum of neonatal rat brain. Neurochem Int 2011;58: 625-633.

28 Hubbard WK: United States Food and Drug Administration. Letter responding to health claim petition dated November 3, 2003 (Martek petition): Omega- 3 fatty acids and reduced risk of coronary heart disease (docket No. 2003Q-0401). Date of issue September 8, 2004. http://www.fda.gov/Food/LabelingNutrition/LabelClaims/QualifiedHealthClaims/ ucm072932.htm (accessed September 30, 2011).

29 Diamond IR, Sterescu A, Pencharz PB, Kim $\mathrm{JH}$, Wales PW: Changing the paradigm: omegaven for the treatment of liver failure in pediatric short bowel syndrome. J Pediatr Gastroenterol Nutr 2009;48:209-215. 